\title{
Percepción del alumnado de máster sobre la formación en competencias emprendedoras a través de redes sociales
}

\author{
Sandra Clemente-Vázquez \\ Juan-Jesús Torres-Gordillo \\ Universidad de Sevilla. España. \\ sandra_97_12@hotmail.com \\ juanj@us.es
}

Recibido: 29/7/2020

Aceptado: 5/2/2021

Publicado: 5/7/2021

\section{Resumen}

El objetivo del presente estudio es analizar la percepción y el origen del nivel competencial en emprendimiento a través de redes sociales en el alumnado de másteres de las ramas de Ciencias, Humanidades y Artes y Ciencias Sociales ofertados por la Universidad de Sevilla. La crisis económica en España ha provocado que el emprendimiento sea, en muchos casos, la única vía de emplearse. Internet y el comercio electrónico obligan a tener en cuenta las redes sociales para acceder al mercado global. Se refuerza la universidad como figura esencial para crear cultura emprendedora a través de la formación de su alumnado. Se asume un diseño de investigación cuantitativo ex post facto, no experimental y de tipo descriptivo, correlacional e inferencial. El estudio se realizó con un muestreo no probabilístico por conveniencia. 178 estudiantes contestaron a un cuestionario ad hoc. Los resultados indican un grado entre moderado y bajo en cuanto a la percepción competencial del alumnado, aunque con algunas diferencias según la edad o el tipo de máster. Se constata que la educación formal superior no satisface al estudiantado en este sentido. Se concluye que la universidad debe mejorar su formación en materia de emprendimiento a través de redes sociales.

Palabras clave: educación y empleo; redes sociales; universidad; formación; competencias para la vida; educación superior

Resum. Percepció de l'alumnat de màster sobre la formació en competències emprenedores a través de xarxes socials

L'objectiu del present estudi és analitzar la percepció i l'origen del nivell competencial en emprenedoria a través de xarxes socials en l'alumnat de màsters de les branques de Ciències, Humanitats i Arts i Ciències Socials oferts per la Universitat de Sevilla. La crisi econòmica a Espanya ha provocat que l'emprenedoria sigui, en molts casos, l'única via per poder treballar. Internet i el comerç electrònic obliguen a tenir en compte les xarxes socials per a accedir al mercat global. Es reforça la universitat com a figura essencial per a crear cultura emprenedora a través de la formació del seu alumnat. S'assumeix un disseny de recerca quantitatiu ex post facto, no experimental i de tipus descriptiu, correlacional i inferencial. L'estudi es va realitzar amb un mostreig no probabilístic per conveniència. 178 
estudiants van contestar a un qüestionari ad hoc. Els resultats indiquen un grau entre moderat i baix quant a la percepció competencial de l'alumnat, encara que amb algunes diferències segons l'edat o el tipus de màster. Es constata que l'educació formal superior no satisfa l'estudiantat en aquest sentit. Es conclou que la universitat ha de millorar la seva formació en matèria d'emprenedoria a través de xarxes socials.

Paraules clau: educació i ocupació; xarxes socials; universitat; formació; competències per a la vida; educació superior

Abstract. Master's Students' Perception of Training in Entrepreneurial Skills Through Social Networks

The aim of this study is to analyze the perception and origin of entrepreneurship competence through social networks among master's degree students in sciences, humanities and arts, and social sciences at the University of Seville, Spain. The economic crisis in Spain has meant that entrepreneurship is often the only way to be employed, while the Internet and e-commerce have obliged us to use social networks to access the global market. Universities play an essential role in creating an entrepreneurial culture through the training of students. A quantitative, ex post facto, non-experimental, descriptive, correlational, and inferential research design was used with non-probabilistic convenience sampling. A total of 178 students completed an ad hoc questionnaire. The results indicate that students perceive their competence level as being moderate to low, although with some differences according to age or type of master's degree. It was observed that formal higher education does not satisfy students' expectations in this regard. Therefore, we conclude that training in entrepreneurship through social networks should be improved in the higher education setting.

Keywords: education and employment; social networks; universities; training; life skills; higher education

\author{
Sumario \\ 1. Introducción 4. Discusión y conclusiones \\ 2. Método Referencias bibliográficas
}

3. Resultados

\title{
1. Introducción
}

Nuestra sociedad líquida, el auge tecnológico y la globalización han provocado cambios en nuestra manera de vivir. Ciertas capacidades se han convertido en obsoletas, lo que ha obligado a realizar una transformación social. Encontramos profesiones cambiantes y unas necesidades educativas diferentes (Barreno, 2011; Sánchez-García, 2017).

El desempleo es la tónica habitual del mercado laboral. Cada vez se apuesta más por el emprendimiento, que toma fuerza en el ámbito académico, económico y político. Es, en muchos casos, la única vía para poder trabajar (Gómez-Núnez et al., 2017; Ortiz-García y Olaz-Capitán, 2015; Trejo-López, 2016). El emprendimiento es una forma de avanzar ante la insatisfacción. Nos 
lleva a comenzar una tarea con mayores objetivos. Pero no solo hace falta tener una idea de negocio, sino que también requiere una serie de habilidades, actitudes y conocimientos que transformen las ideas en acción (European Commission, 2016; Gutiérrez-Olvera, 2015; Morales y Corredor, 2016; PalosSánchez, Baena-Luna y Casablanca-Peña, 2019).

\subsection{Emprendimiento a través de las redes sociales}

Actualmente Internet y el comercio electrónico deben formar parte del negocio (Becerra-Molina, Sañay y Calle, 2018; Rodríguez-Acosta, 2018). Es necesario atraer a un público, y es ahí donde las redes sociales entran en juego (Estévez- Gualda y García-Marín, 2015). Las redes sociales son espacios formados por comunidades virtuales a través de la web 2.0. Son herramientas de comunicación útiles para la visibilidad del negocio, incluso más que otros medios de comunicación tradicionales (Fernández-Trigueros y FernándezCastaño, 2014; Fuster-Gullén et al., 2020; Peñarroya-Farell, 2014). El objetivo es mostrar un producto o servicio que capte a un público consumidor, creándose una conexión entre clientela y empresa (Castro-Villacis, RodríguezFigueroa, Arroyo-Vargas y Valdés-Cabrera, 2018).

Surgen entonces una serie de cuestiones: ¿qué competencias se necesitan para emprender en la actualidad? ¿Son innatas o se aprenden?

\subsection{Formación en competencias emprendedoras y redes sociales}

Según el marco de referencia europeo de la competencia emprendedora EntreComp, aprobado por la Comisión Europea en 2016 (Bacigalupo, Kampylis, Punie y Van den Brande, 2016), encontramos tres áreas que clasifican las diferentes subcompetencias de la persona emprendedora (Estrada de la Cruz, Mira-Solves y Gómez-Gras, 2016; Jiménez-Marín, Elías-Zambrano, y SilvaRobles, 2014; Morales y Corredor, 2016): ideas y oportunidades, recursos y pasar a la acción (ver la tabla 1 ).

Muchas personas emprendedoras adquirieron estos conocimientos a través de la experiencia laboral previa a la creación del negocio. Trayectorias educativas demuestran que estas competencias se enseñan y se aprenden (CivilaSalas, 2017; Jiménez-Marín, Elías-Zambrano y Silva-Robles, 2014; JustinianoDomínguez, 2019).

La educación para el emprendimiento se entiende como el desarrollo del conocimiento empresarial, las habilidades y las actitudes empresariales (Oleforo-Ngozika, Oko-Dominic y Akpan, 2013; Zenner, Kothandaraman y Pilz, 2017). Sin embargo, solo el $9,8 \%$ de la población joven encuestada ha emprendido alguna actividad o se encuentra en vías de hacerlo (Ortiz-García y OlazCapitán, 2015).

Con la Ley de Emprendedores (Ley 11/2013 de 26 de julio), España pretende contribuir al estímulo del emprendimiento en población joven (Fernández-Trigueros y Fernández-Castaño, 2014). Es uno de los objetivos de la Ley 
Tabla 1. Áreas y subcompetencias emprendedoras

\begin{tabular}{|c|c|c|}
\hline $\begin{array}{l}\text { Área 1: } \\
\text { Ideas y oportunidades }\end{array}$ & $\begin{array}{l}\text { Área 2: } \\
\text { Recursos }\end{array}$ & $\begin{array}{l}\text { Área 3: } \\
\text { Pasar a la acción }\end{array}$ \\
\hline Pensamiento ético y sostenible & $\begin{array}{l}\text { Autoconocimiento y confianza } \\
\text { en uno mismo }\end{array}$ & $\begin{array}{l}\text { Manejo de la incertidumbre } \\
\text { y el riesgo }\end{array}$ \\
\hline Identificación de oportunidades & Perseverancia & Aprendizaje de la experiencia \\
\hline Valoración de ideas & $\begin{array}{l}\text { Educación financiera y eco- } \\
\text { nómica }\end{array}$ & Planificación y gestión \\
\hline Creatividad & Movilización de recursos & Trabajo con otros \\
\hline Visión & Involucramiento a otros & Iniciativa y toma de decisiones \\
\hline Innovación & Liderazgo y comunicación & $\begin{array}{l}\text { Adaptación a entornos cam- } \\
\text { biantes }\end{array}$ \\
\hline $\begin{array}{l}\text { Gestión de la innovación dentro } \\
\text { de la empresa }\end{array}$ & Motivación & Creación de valor \\
\hline
\end{tabular}

Fuente: elaboración propia.

Orgánica 8/2013, de 9 de diciembre, para la Mejora de la Calidad Educativa, entendiéndose este espíritu como el impulso del individuo con ambición y oportunidades para tomar riesgos e iniciar su propio negocio (Alaref, Brodmann y Premand, 2019).

Dicha formación emprendedora del currículum de la enseñanza pública debe acompañarse del aprendizaje del manejo y aprovechamiento de las tecnologías, haciendo un uso transversal y eficaz (Erdoğmuş, Korkmaz, Çakir y Uğur-erdoğmuş, 2019). Al analizar la autoeficacia emprendedora a través de la tecnología, los resultados no superan la mitad de la escala propuesta en el estudio (Torres-Coronas, Vidal-Blasco y Arias-Oliva, 2014). Sin embargo, tras la formación en emprendimiento mediante redes sociales, se ha conseguido aumentar de un 9\% a más del 20\% de estudiantes capaces de aprovechar estas plataformas para lanzar un proyecto profesional (Estévez-Gualda y GarcíaMarín, 2015).

\subsection{Emprendimiento, redes sociales y universidad}

El uso de la tecnología se ha incrementado en los últimos años dentro de las universidades. Además, el profesorado universitario es consciente de los beneficios que aporta el trabajo por competencias al aprendizaje del alumnado (Fernández-Ferrer y Forés-Miravalles, 2018). Es una buena ocasión para aprovecharlo en materia de emprendimiento (Calderón-Garrido, León-Gómez y Gil-Fernández, 2019; Mese y Aydin, 2019; Mirembe, Lubega y Kibukamusoke, 2019).

La mayoría de las universidades públicas españolas llevan a cabo programas, cursos y talleres dentro del currículo de apoyo al emprendimiento, aunque en algunos casos son opcionales para el alumnado. Estos cursos están orientados a la elaboración de planes de negocio, finanzas, marketing o leyes. 
Dejan de lado las redes sociales o los recursos individuales, y competencias como la selección de socios y socias, la conformación de equipos y de alianzas, el contacto con clientela y personas inversoras, etc. (Torres-Briones et al., 2018). Puede verse en los planes de estudio de carreras como Turismo, Publicidad y Relaciones Públicas o Administración y Dirección de Empresas. Carreras alejadas del enfoque emprendedor, como el grado en Maestro/a, en Pedagogía o en Ciencias de la Actividad Física y del Deporte, no cuentan con asignaturas de carácter emprendedor.

Solo aquellos másteres dirigidos exclusivamente al emprendimiento ofrecen alguna asignatura sobre redes sociales y creación de empresas. El informe GEM (2018) indica que el 49,8\% de las personas emprendedoras en fase inicial tienen educación superior o de postgrado, frente al 50,2\% que posee estudios primarios o secundarios. La formación universitaria juega un papel fundamental en la creación de esta cultura emprendedora. Puede aprovechar su experiencia en la educación aplicada para desarrollar una política de emprendimiento que favorezca a la viabilidad económica y al éxito de sus comunidades (Menna, Catalfamo y Girolamo, 2016).

El informe GEM de España (2018) recomienda, para mejorar el emprendimiento, más calidad y cantidad de programas de creación de empresas en todos los niveles educativos, promoviendo las competencias y los valores, más concretamente, en la etapa universitaria. Una formación en emprendimiento a través de redes sociales desarrollaría las habilidades del estudiantado para afrontar y confiar en su carrera profesional, lo que incrementaría sus intenciones empresariales (Qiao y Hua, 2019).

Con todo ello, surge la pregunta de investigación: ¿se siente preparado el estudiantado de máster para emprender a través de las redes sociales? Los objetivos derivados de esta cuestión son conocer la percepción y el origen del nivel competencial.

\section{Método}

\subsection{Diseño de investigación}

Se asume un diseño de investigación cuantitativo ex post facto, no experimental, de carácter descriptivo, correlacional e inferencial. El objetivo es conocer las percepciones del alumnado de máster sobre su nivel competencial en cuanto a emprendimiento en redes sociales, así como el origen de estas (innato, experiencia profesional, educación formal superior o no formal). La tabla 2 recoge el problema, el objetivo y las variables de investigación. 
Tabla 2. Problema, objetivos y variables de investigación

\begin{tabular}{ll}
\hline $\begin{array}{l}\text { Problema de investigación: ¿se siente preparado el estudiantado de máster para emprender } \\
\text { a través de las redes sociales? }\end{array}$ & Variables \\
\hline Objetivos & Percepción del nivel competencial. \\
\hline $\begin{array}{l}\text { 1. Conocer la percepción sobre el grado de } \\
\text { adquisición de las competencias empren- } \\
\text { dedoras aplicadas a las redes sociales del } \\
\text { estudiantado de máster. }\end{array}$ & \\
$\begin{array}{l}\text { 2. Conocer el origen (innato, experiencial o } \\
\text { formativo) de las competencias emprende- } \\
\text { doras de la población encuestada. }\end{array}$ & Origen de competencias. \\
\hline
\end{tabular}

Fuente: elaboración propia.

\subsection{Selección de los participantes}

Se aplica un cuestionario en línea ad hoc basado en las áreas y en las subcompetencias de la persona emprendedora, recogidas por el Marco de referencia europeo de la competencia emprendedora (Bacigalupo, Kampylis, Punie y Van den Brande, 2016) y otros estudios (Estrada de la Cruz, Mira-Solves y GómezGras, 2016; Jiménez-Marín, Elías-Zambrano, y Silva-Robles, 2014; Morales y Corredor, 2016). La muestra es no probabilística por conveniencia de alumnado de másteres oficiales del curso 2019-2020 ofertados por la Universidad de Sevilla de las ramas de conocimiento de Ciencias, Humanidades y Artes y Ciencias Sociales. Se obtuvieron respuestas de 178 estudiantes de entre 22 y 55 años, con un $\pm 6,99 \%$ de error muestral (ver la tabla 3 ).

Tabla 3. Participantes de la investigación según la rama de conocimiento y el grupo de edad

\begin{tabular}{lc}
\hline Ramas de conocimiento de los másteres evaluados & Participantes \\
\hline Ciencias & 53 \\
Humanidades y Artes & 58 \\
Ciencias Sociales & 67 \\
\hline Grupos de edad & Participantes \\
\hline Hasta 25 años & 92 \\
\hline A partir de 26 años & 86 \\
\hline
\end{tabular}

Fuente: elaboración propia.

\subsection{Instrumento de recogida de datos}

El instrumento es un cuestionario en línea, estructurado en dos variables (Percepción del nivel competencial y Origen de competencias) con 24 ítems. Se administró sobre los 178 sujetos utilizando la herramienta en línea Formularios de Google. Las respuestas se organizan en una escala de tipo Likert de 5 puntos para cada dimensión (ver la tabla 4). 
Tabla 4. Escala Likert según la dimensión

\begin{tabular}{|c|c|}
\hline Percepción del nivel competencial & Origen de competencias \\
\hline 1. No la poseo (NP) & 1. Totalmente en desacuerdo \\
\hline 2. La poseo en grado bajo (B) & 2. En desacuerdo \\
\hline 3. La poseo en un grado intermedio (I) & 3. Neutro \\
\hline 4. La poseo en un grado alto $(A)$ & 4. De acuerdo \\
\hline 5. La poseo completamente (C) & 5. Totalmente de acuerdo \\
\hline
\end{tabular}

Fuente: elaboración propia.

La dimensión Percepción del nivel competencial se refiere al grado en que el alumnado se siente poseedor de las competencias emprendedoras aplicadas a las redes sociales. La dimensión Origen de competencias representa el grado en que la competencia ha sido adquirida de manera innata, como resultado de la experiencia profesional, o bien como aprendizaje adquirido a través de la educación formal en la etapa superior o en la educación no formal.

Se calcularon los valores alfa de Cronbach para estimar la fiabilidad del instrumento en su conjunto y de cada dimensión considerada. La fiabilidad, según su consistencia interna, es excelente, exhibiendo un valor de 0,94. Además, se llevó a cabo la validación mediante la técnica del juicio de expertos. Se contactó con 9 expertos de grupos de investigación de Ciencias de la Educación, Psicología y Comunicación de la Universidad de Sevilla. Propusieron añadir o quitar ciertos ítems y realizar cambios en la redacción del contenido. Se aseguró el consentimiento informado.

\subsection{Análisis de datos}

Los datos se organizaron atendiendo a las tres ramas de conocimiento de los másteres y los dos grupos de edad estudiados, y se analizaron con el software SPSS V.23. Se realizaron análisis descriptivos, correlaciones y contrastes. En el descriptivo se consideró la media y la desviación típica de los ítems.

Para averiguar si existían correlaciones entre las variables ordinales, se realizó la correlación de Spearman. Se aplicó el coeficiente de contingencia para conocer la relación entre las variables nominales Tipo de máster y Edad.

Para saber si existe relación entre estas dos variables, se realizaron pruebas de contraste no paramétricas. Previo a este proceso se ejecutó la prueba K-S para comprobar el supuesto de normalidad. Al no cumplirse, atendiendo a la variable Edad, se efectuó la prueba U de Mann-Whitney para conocer si existían diferencias significativas entre los dos grupos.

Respecto al tipo de máster, se aplicó la prueba $\mathrm{H}$ de Kruskal-Wallis. Para detectar falsos positivos o negativos en las pruebas de contraste realizadas, se calculó la magnitud del tamaño del efecto a través de la $d$ de Cohen. 


\section{Resultados}

Se presenta el análisis descriptivo de las dos dimensiones (ver la tabla 5). La dimensión Percepción del nivel competencial obtiene una media entre intermedia y baja (no supera el valor $M=3,5$ ). Esto refleja ciertas carencias percibidas a nivel competencial en lo que respecta al emprendimiento en redes sociales. La desviación típica de los ítems es superior a 1, dándose la mayor desviación en el ítem $6(\mathrm{DT}=1,280)$, y la menor, en el ítem $20(\mathrm{DT}=1,033)$. Aquellas competencias que el estudiantado cree poseer en menor medida son el ítem $11(\mathrm{M}=2,27$; $\mathrm{DT}=1,196)$; el $2(\mathrm{M}=2,44 ; \mathrm{DT}=1,188)$ y el $7(\mathrm{M}=2,49$; $\mathrm{DT}=1,136)$. Las competencias que creen poseer en mayor medida son el ítem $15(\mathrm{M}=3,55$; $\mathrm{DT}=1,179)$; el $17(\mathrm{M}=3,53$; $\mathrm{DT}=1,175)$ y el $14(\mathrm{M}=3,47$; $\mathrm{DT}=1,203)$. Dichos ítems hacen referencia a competencias menos relacionadas con capacidades técnicas.

En la dimensión Origen de competencias el estudiantado siente que lo aprendido en emprendimiento mediante redes sociales proviene, en buena parte, de la experiencia profesional $(\mathrm{M}=3,54 ; \mathrm{DT}=1,231)$ o de la educación no formal $(\mathrm{M}=3,10 ; \mathrm{DT}=1,198)$. El alumnado no considera innatas sus competencias $(\mathrm{M}=2,88 ; \mathrm{DT}=1,172)$, ni especialmente que provengan de la educación superior $(\mathrm{M}=2,55$; $\mathrm{DT}=1,198)$.

Tabla 5. Análisis descriptivo de los ítems

\begin{tabular}{|c|c|c|}
\hline & M & DT \\
\hline 1. Usar RRSS para emprender & 2,67 & 1,167 \\
\hline 2. Crear negocio en RRSS con responsabilidad social & 2,44 & 1,188 \\
\hline 3. Identificar una oportunidad provechosa para crear negocio RRSS & 2,58 & 1,133 \\
\hline 4. Evaluar ideas de negocio & 2,85 & 1,189 \\
\hline 5. Manifestar creatividad en el proceso de crear negocio en RRSS & 3,13 & 1,255 \\
\hline 6. Tener visión estratégica en negocio RRSS & 2,69 & 1,280 \\
\hline 7. Crear negocio que genere novedad en RRSS & 2,49 & 1,136 \\
\hline $\begin{array}{l}\text { 8. Gestionar recursos de empresa para crear nuevos conceptos y/o productos } \\
\text { innovadores }\end{array}$ & 2,73 & 1,157 \\
\hline 9. Confiar en mí mismo al llevar el negocio en RRSS & 3,13 & 1,259 \\
\hline 10. Ser perseverante, avanzar a pesar de los obstáculos en el negocio & 3,34 & 1,226 \\
\hline 11. Aplicar conocimientos financieros y económicos en el negocio & 2,27 & 1,196 \\
\hline $\begin{array}{l}\text { 12. Crear lazos y contactos con otras personas para beneficiar al negocio en } \\
\text { RRSS }\end{array}$ & 3,17 & 1,178 \\
\hline $\begin{array}{l}\text { 13. Liderar el equipo de trabajo tomando decisiones de negocio, organizando } \\
\text { tareas de los demás }\end{array}$ & 3,26 & 1,244 \\
\hline 14. Enganchar al equipo y conectar con él para que se ilusione con el proyecto & 3,47 & 1,203 \\
\hline 15. Mantener la motivación del equipo de negocio & 3,55 & 1,179 \\
\hline 16. Manejar de forma positiva el riesgo al lanzar el proyecto profesional en RRSS & 2,92 & 1,205 \\
\hline
\end{tabular}


17. Aprender de experiencias del proceso de negocio en RRSS para no repetir errores

18. Planificar y gestionar todas las fases del proceso de creación de negocio en RRSS

19. Adaptarme a distintos escenarios y contextos cambiantes durante el proceso de negocio en RRSS

20. Generar ganancia y utilidad a través de la actividad emprendedora en RRSS

21. Generar ganancia y utilidad a través de la educación formal recibida en la formación superior (grado y máster)

22. Generar ganancia y utilidad a través de la educación no formal (cursos, talleres, etc.)

23. Generar ganancia y utilidad a través de la experiencia profesional

Fuente: elaboración propia.

Las correlaciones indicaron resultados significativos $(p<0,05)$ en la dimensión Percepción del nivel competencial (ver las tablas 6 y 7). Se muestran solo aquellas correlaciones con un coeficiente alto y significación al 0,000. Con un $99 \%$ de confianza. Conforme aumenta la percepción del nivel de una competencia, existe un aumento en la percepción de la mayoría del resto.

Tabla 6. Análisis de correlaciones empleando el coeficiente de Spearman

\begin{tabular}{|c|c|c|c|c|c|c|c|c|c|c|}
\hline Coef. & p1. & p2. & p3. & p4. & p5. & p6. & p7. & p8. & p9. & p10. \\
\hline p1. & & $0,685^{\star *}$ & & & & & & & & \\
\hline p2. & $0,648^{\star \star}$ & $0,654^{\star *}$ & & & & & & & & \\
\hline p3. & & & $0,728^{\star *}$ & & & & & & & \\
\hline p4. & $0,645^{\star \star}$ & & $0,601^{* *}$ & $0,609^{* *}$ & & & & & & \\
\hline p5. & $0,654^{\star *}$ & $0,624^{\star *}$ & $0,725^{\star *}$ & $0,700^{\star *}$ & $0,649^{* *}$ & & & & & \\
\hline p6. & $0,641^{* *}$ & & $0,720^{* *}$ & $0,650^{* *}$ & $0,668^{\star *}$ & $0,744^{* *}$ & & & & \\
\hline p7. & & & $0,640^{* *}$ & $0,693^{* *}$ & $0,620^{* *}$ & $0,685^{\star *}$ & $0,792^{* *}$ & & & \\
\hline p8. & & & $0,654^{* *}$ & & & $0,633^{* *}$ & & $0,610^{\star *}$ & & \\
\hline p9. & & & $0,563^{\star *}$ & $0,564^{\star \star}$ & & $0,605^{\star *}$ & & & $0,732^{\star *}$ & \\
\hline p11. & & & & & & & & $0,612^{* *}$ & & $0,602^{* *}$ \\
\hline p12. & & & & $0,616^{* *}$ & & $0,623^{\star *}$ & & $0,637^{\star \star}$ & & $0,630^{* *}$ \\
\hline p13. & & & & & & & $0,624^{\star *}$ & $0,673^{\star \star}$ & & $0,647^{\star \star}$ \\
\hline p14. & & & & & & & & & & $0,620^{\star \star}$ \\
\hline p15. & & & & & & $0,638^{\star *}$ & $0,651^{* *}$ & $0,604^{\star *}$ & $0,649^{* *}$ & $0,663^{* *}$ \\
\hline p16. & & & & & & & & & & $0,634^{\star *}$ \\
\hline p17. & & & & & & & & & & $0,609^{* *}$ \\
\hline p18. & & & & & & & & & $0,642^{\star *}$ & $0,612^{* *}$ \\
\hline p19. & & & $0,619^{\star *}$ & $0,602^{* *}$ & & $0,617^{\star *}$ & & $0,616^{\star \star}$ & $0,691^{* *}$ & \\
\hline
\end{tabular}

Fuente: elaboración propia. 
Tabla 7. Análisis de correlaciones empleando el coeficiente de Spearman

\begin{tabular}{|c|c|c|c|c|c|c|c|c|c|c|}
\hline & Coef. p11. & p12. & p13. & p14. & p15. & p16. & p17. & p18. & p19. & p20. \\
\hline p2. & & & & & & & & & & $0,619^{* *}$ \\
\hline p3. & & & $0,616^{* *}$ & & & & & & & $0,602^{\star *}$ \\
\hline p5. & & & $0,623^{* *}$ & & & $0,638^{\star *}$ & & & & $0,617^{\star \star}$ \\
\hline p6. & & & & $0,624^{\star *}$ & & $0,651^{* *}$ & & & & \\
\hline p7. & & $0,612^{\star *}$ & $0,637^{* *}$ & $0,673^{\star \star}$ & & $0,604^{\star *}$ & & & & $0,616^{\star \star}$ \\
\hline p8. & & & & & & $0,649^{* *}$ & & & $0,642^{* *}$ & $0,691^{* *}$ \\
\hline p9. & & $0,602^{\star *}$ & $0,630^{* *}$ & $0,647^{\star \star}$ & $0,620^{* \star}$ & $0,663^{\star *}$ & $0,634^{\star *}$ & $0,609^{* *}$ & $0,612^{\star *}$ & \\
\hline p11. & & & & $0,601^{* *}$ & & & $0,638^{* *}$ & $0,613^{* *}$ & $0,608^{* *}$ & \\
\hline \multicolumn{11}{|l|}{ p12. } \\
\hline p13. & & $0,601^{\star *}$ & $0,758^{* *}$ & & & & & & & \\
\hline p14. & & & $0,683^{* *}$ & $0,785^{\star *}$ & & & & & & \\
\hline p15. & & & & $0,618^{* *}$ & $0,603^{* *}$ & & & & & \\
\hline p16. & & $0,638^{\star \star}$ & $0,603^{* *}$ & $0,666^{\star \star}$ & $0,657^{\star \star}$ & $0,639^{\star *}$ & & & & \\
\hline p17. & & $0,613^{\star *}$ & & $0,603^{\star *}$ & $0,644^{\star \star}$ & $0,670^{* *}$ & $0,707^{\star *}$ & & & \\
\hline p18. & & $0,608^{\star *}$ & & & & $0,666^{* *}$ & $0,644^{* *}$ & $0,659^{* *}$ & & \\
\hline p19. & & & & & & & & $0,620^{* *}$ & $0,654^{\star *}$ & \\
\hline
\end{tabular}

Fuente: elaboración propia.

Con un 95\% de confianza, aunque con una relación baja $(0,239)$, la tendencia es que hasta 25 años el origen de las competencias no viene por la educación superior. Los mayores de 25 años concentran sus opiniones por igual entre el desacuerdo y el acuerdo, pasando por una respuesta neutra (ver la tabla 8). Con un coeficiente bajo $(0,291)$, el grupo de hasta 25 años no considera la educación no formal como el origen de sus competencias. Los mayores de 25 años sí la consideran así (ver la tabla 9). Con un coeficiente bajo $(0,234)$, la tendencia es que hasta los 25 años el origen no está en la experiencia profesional (salvo excepciones). Los mayores de 25 años se posicionan entre la neutralidad y estar muy de acuerdo con que el origen de las competencias viene por la experiencia profesional (ver la tabla 10). 
Tabla 8. Percepción del origen de las competencias a través de la educación formal superior según la edad

\begin{tabular}{|c|c|c|c|c|c|c|c|}
\hline & & & \multicolumn{2}{|c|}{ Edad } & \multirow[t]{2}{*}{ Total } & \multirow{2}{*}{$\begin{array}{l}\text { Coef. } \\
\text { conting. }\end{array}$} & \multirow[t]{2}{*}{ Sig. } \\
\hline & & & $<25$ & $>25$ & & & \\
\hline \multirow[t]{10}{*}{ p21. } & TD & Recuento & 29 & 18 & 47 & \multirow{10}{*}{0,239} & \multirow{10}{*}{0,029} \\
\hline & & $\begin{array}{l}\text { Frecuencia esperada } \\
\text { (FE) }\end{array}$ & 24,3 & 22,7 & 47,0 & & \\
\hline & \multirow[t]{2}{*}{ ED } & Recuento & 18 & 19 & 37 & & \\
\hline & & FE & 19,1 & 17,9 & 37,0 & & \\
\hline & \multirow[t]{2}{*}{$\mathrm{N}$} & Recuento & 22 & 28 & 50 & & \\
\hline & & FE & 25,8 & 24,2 & 50,0 & & \\
\hline & \multirow[t]{2}{*}{$\mathrm{DA}$} & Recuento & 16 & 21 & 37 & & \\
\hline & & $\mathrm{FE}$ & 19,1 & 17,9 & 37,0 & & \\
\hline & \multirow[t]{2}{*}{$\mathrm{TA}$} & Recuento & 7 & 0 & 7 & & \\
\hline & & $\mathrm{FE}$ & 3,6 & 3,4 & 7,0 & & \\
\hline
\end{tabular}

Fuente: elaboración propia.

Tabla 9. Percepción del origen de competencias a través de la educación no formal según la edad

\begin{tabular}{|c|c|c|c|c|c|c|c|}
\hline & & & \multicolumn{2}{|c|}{ Edad } & \multirow[t]{2}{*}{ Total } & \multirow{2}{*}{$\begin{array}{c}\text { Coef. } \\
\text { conting. }\end{array}$} & \multirow[t]{2}{*}{ Sig. } \\
\hline & & & $<25$ & $>25$ & & & \\
\hline \multirow[t]{10}{*}{ p22. } & TD & Recuento & 19 & 6 & 25 & \multirow{10}{*}{0,291} & \multirow{10}{*}{0,002} \\
\hline & & FE & 12,9 & 12,1 & 25,0 & & \\
\hline & \multirow[t]{2}{*}{ ED } & Recuento & 17 & 10 & 27 & & \\
\hline & & FE & 14,0 & 13,0 & 27,0 & & \\
\hline & \multirow[t]{2}{*}{$\mathrm{N}$} & Recuento & 27 & 21 & 48 & & \\
\hline & & $\mathrm{FE}$ & 24,8 & 23,2 & 48,0 & & \\
\hline & \multirow[t]{2}{*}{$\mathrm{DA}$} & Recuento & 20 & 41 & 61 & & \\
\hline & & $\mathrm{FE}$ & 31,5 & 29,5 & 61,0 & & \\
\hline & \multirow[t]{2}{*}{ TD } & Recuento & 9 & 8 & 17 & & \\
\hline & & $\mathrm{FE}$ & 8,8 & 8,2 & 17,0 & & \\
\hline
\end{tabular}

Fuente: elaboración propia. 
Tabla 10. Percepción del origen de competencias a través de la experiencia profesional según la edad

\begin{tabular}{|c|c|c|c|c|c|c|c|}
\hline & & & \multicolumn{2}{|c|}{ Edad } & \multirow[t]{2}{*}{ Total } & \multirow{2}{*}{$\begin{array}{c}\text { Coef. } \\
\text { conting. }\end{array}$} & \multirow[t]{2}{*}{ Sig. } \\
\hline & & & $<25$ & $>25$ & & & \\
\hline \multirow[t]{10}{*}{ p23. } & \multirow[t]{2}{*}{ TD } & Recuento & 13 & 4 & 17 & & \\
\hline & & $\mathrm{FE}$ & 8,8 & 8,2 & 17,0 & & \\
\hline & \multirow[t]{2}{*}{ ED } & Recuento & 11 & 4 & 15 & & \\
\hline & & $\mathrm{FE}$ & 7,8 & 7,2 & 15,0 & & \\
\hline & \multirow[t]{2}{*}{$\mathrm{N}$} & Recuento & 20 & 26 & 46 & 0,234 & 0,036 \\
\hline & & $\mathrm{FE}$ & 23,8 & 22,2 & 46,0 & & \\
\hline & \multirow[t]{2}{*}{ DA } & Recuento & 29 & 25 & 54 & & \\
\hline & & $\mathrm{FE}$ & 27,9 & 26,1 & 54,0 & & \\
\hline & \multirow[t]{2}{*}{$\mathrm{TA}$} & Recuento & 19 & 27 & 46 & & \\
\hline & & $\mathrm{FE}$ & 23,8 & 22,2 & 46,0 & & \\
\hline
\end{tabular}

Fuente: elaboración propia.

Según el tipo de máster, con un coeficiente bajo $(0,289)$, podemos decir que la percepción competencial de Mantener motivación del equipo de negocio la posee completamente una parte del estudiantado de másteres de Humanidades y Artes, aunque hay otro porcentaje menor que no la posee o solo en grado medio. El alumnado de Ciencias la posee en un grado alto, y el de Ciencias Sociales la posee en grado medio (ver la tabla 11).

Tabla 11. Percepción sobre el grado de adquisición de las competencias según el tipo de máster

\begin{tabular}{|c|c|c|c|c|c|c|c|c|}
\hline & & & \multicolumn{3}{|c|}{ Máster } & \multirow[t]{2}{*}{ Total } & \multirow{2}{*}{$\begin{array}{c}\text { Coef. } \\
\text { conting. }\end{array}$} & \multirow[t]{2}{*}{ Sig. } \\
\hline & & & $\begin{array}{l}\text { Humanidades } \\
\text { y Artes }\end{array}$ & Ciencias & $\begin{array}{l}\text { Ciencias } \\
\text { Sociales }\end{array}$ & & & \\
\hline \multirow[t]{10}{*}{ p15. } & NP & Recuento & 8 & 5 & 4 & 17 & \multirow{10}{*}{0,289} & \multirow{10}{*}{0,035} \\
\hline & & $\mathrm{FE}$ & 5,5 & 5,1 & 6,4 & 17,0 & & \\
\hline & \multirow[t]{2}{*}{ B } & Recuento & 4 & 3 & 7 & 14 & & \\
\hline & & $\mathrm{FE}$ & 4,6 & 4,2 & 5,3 & 14,0 & & \\
\hline & \multirow[t]{2}{*}{ I } & Recuento & 14 & 5 & 18 & 37 & & \\
\hline & & $\mathrm{FE}$ & 12,1 & 11,0 & 13,9 & 37,0 & & \\
\hline & \multirow[t]{2}{*}{ A } & Recuento & 17 & 32 & 25 & 74 & & \\
\hline & & FE & 24,1 & 22,0 & 27,9 & 74,0 & & \\
\hline & \multirow[t]{2}{*}{ C } & Recuento & 15 & 8 & 13 & 36 & & \\
\hline & & FE & 11,7 & 10,7 & 13,6 & 36,0 & & \\
\hline
\end{tabular}

Fuente: elaboración propia. 
Tras aplicar las pruebas de contraste no paramétricas mediante la prueba U de Mann-Whitney (ver la tabla 12), observamos que existen diferencias significativas, con un nivel de confianza del $99 \%$, en ser mayor de 25 años y tener el origen competencial en la educación no formal, así como tenerlo por experiencia profesional (confianza del 95\%). Atendiendo a la magnitud del tamaño del efecto, según la $d$ de Cohen, se consideran diferencias que son de intensidad moderada en el ítem $22(d=0,5)$ y de intensidad baja en el ítem $23(d=0,3)$.

Tabla 12. Prueba de $U$ de Mann-Whitney según la edad

\begin{tabular}{ccccccc}
\hline & Edad & $\boldsymbol{N}$ & Rango promedio & U de Mann-Whitney & Sig. (bilateral) & $\boldsymbol{d}$ \\
\hline \multirow{2}{*}{ p22. } & $<25$ & 92 & 77,45 & 2847,500 & 0,001 & 0,5 \\
& $>25$ & 86 & 102,39 & & & \\
\multirow{2}{*}{ p23. } & $<25$ & 92 & 81,70 & 3238,000 & 0,031 & 0,3 \\
& $>25$ & 86 & 97,85 & & & \\
\hline
\end{tabular}

Fuente: elaboración propia.

Con respecto al tipo de máster, podemos afirmar, con un nivel de confianza del 95\%, que el estudiantado de Ciencias Sociales percibe la competencia de Aplicar conocimientos financieros y económicos en el propio negocio en mayor medida que, al menos, el alumnado de Ciencias y Humanidades y Artes (ver la tabla 13). Atendiendo a la magnitud del tamaño del efecto, se considera una diferencia de intensidad moderada $(d=0,4)$.

Tabla 13. Prueba $\mathrm{H}$ de Kruskal-Wallis según el tipo de máster

\begin{tabular}{llccccc}
\hline \multicolumn{1}{c}{ Tipo de máster } & $\boldsymbol{N}$ & Rango prom. & Chi-cuadrado & Sig. (bilateral) & $\boldsymbol{d}$ \\
\hline p11. & Humanidades y Artes & 58 & 76,82 & & & \\
& Ciencias & 53 & 89,54 & 6,603 & 0,037 & 0,4 \\
& Ciencias Sociales & 67 & 101,03 & & & \\
\hline
\end{tabular}

Fuente: elaboración propia.

\section{Discusión y conclusiones}

Los resultados recogidos reflejan un nivel competencial que no supera el valor $M=3,5$ en el estudiantado. Este resultado es similar a los datos obtenidos por Torres-Coronas, Vidal-Blasco y Arias-Oliva (2014) tras analizar la autoeficacia emprendedora, que no supera la mitad de la escala propuesta.

Respondiendo a nuestra pregunta de investigación, el alumnado de máster se siente poco capaz de emprender en redes sociales. Esto coincide con el análisis de la actividad emprendedora desarrollado por Ortiz-García y OlazCapitán (2015). Solo el 9,8\% de la población joven encuestada había empren- 
dido alguna actividad o se encontraba en vías de hacerlo. Se demostraba la escasa intención emprendedora de la juventud. Atendiendo a nuestros resultados, este hecho podría relacionarse con la falta de competencias emprendedoras.

El alumnado demuestra menos capacidad para aplicar conocimientos financieros y económicos en el negocio, aunque en Ciencias Sociales se siente más preparado para llevar a cabo esta competencia. Esto se da, probablemente, porque es una rama familiarizada con estos conceptos. Pero, al dejar de lado el uso de las redes sociales y otras competencias esenciales (Torres-Briones et al., 2018), hace que no perciba estar preparado para emprender en redes sociales.

Reflejan mayor control en los aspectos relacionados con el área 2 (Recursos) de las subcompetencias emprendedoras marcadas en el marco de referencia europeo EntreComp. Destacan por ser recursos personales, más que competencias técnicas, que podrían aprenderse a través de la educación formal.

La competencia Mantener la motivación del equipo de negocio tiene resultados intermedios y altos. Se alcanzaron mejores resultados en Humanidades y Artes y Ciencias Sociales en cuanto a la adquisición completa de la competencia, aunque Ciencias obtuvo resultados positivos en un grado alto. Podría deberse a que Humanidades y Artes y Ciencias Sociales suelen estar más relacionadas con el trato humano que la rama de Ciencias.

En cuanto al Origen de competencias, la mayor media es para la experiencia profesional en los mayores de 25 años. Las pruebas de contingencia mostraron que hasta los 25 años no consideran la educación no formal ni la experiencia profesional como el origen de sus competencias. Podría estar relacionado con que, a mayor edad, se ha tenido más probabilidad de haber adquirido una mayor experiencia profesional y en la educación no formal. Por último, el origen innato o por la educación superior recibieron las medias más bajas. Los resultados reflejan que la experiencia laboral tiene más peso que la propia capacidad innata, tal y como demuestran otros estudios (Civila-Salas, 2017; Jiménez-Marín, Elías-Zambrano y Silva-Robles, 2014).

Los resultados de nuestro estudio hacen pensar que la formación recibida en los másteres encuestados no resulta suficiente para adquirir estas competencias. Lo poco que el alumnado sabe sobre emprendimiento en redes sociales lo ha aprendido gracias a su experiencia laboral o a la educación no formal. Queda evidenciada la necesidad de mejora por parte de la universidad en materia de emprendimiento. La educación superior debe ser un espacio resonante que genere a personas líderes a través de una formación más vinculada al ámbito profesional. Los estudiantes deben poder descubrir su elemento desde la afección y la emoción que hagan de ellos personas autónomas, exploradoras de sus capacidades y útiles para la vida (Herrero-Vázquez y Torres-Gordillo, 2020).

Se considera importante tener en cuenta las recomendaciones del informe GEM de España (2018) para la mejora del emprendimiento, optando por más calidad y cantidad de programas de creación de empresas aplicables a todos los niveles educativos y promoviendo las competencias y los valores, más concretamente en la etapa universitaria. Todo ello debe ir acompañado de una formación teórica y práctica en competencias digitales desde el inicio de la 
etapa universitaria, que permita el desarrollo del estudiantado en su futuro ámbito profesional (Albertos, Domingo y Albertos, 2016). Para alcanzar este objetivo no podemos seguir trabajando desde la estructura tradicional, donde la docencia es el centro de la enseñanza. Necesitamos metodologías innovadoras que asignen al alumnado universitario un papel activo y adaptado a su estilo de aprendizaje. Es fundamental darle un espacio de formación íntegro y comprometido con la sociedad, donde pueda dar soluciones vinculadas a contextos reales (Melero-Aguilar, Torres-Gordillo y García-Jiménez, 2020; Torres-Gordillo y Herrero-Vázquez, 2020; Torres-Gordillo, Melero-Aguilar y García-Jiménez, 2020). Dentro de estas nuevas metodologías debe tener cabida el uso de la tecnología como herramienta de aprendizaje. Con ello estaremos contribuyendo a la creación de redes de innovación docente, lo que hace que el alumnado acabe conectando más con la asignatura, el profesorado y, especialmente, con el mundo real, aumentando así su nivel de satisfacción (Torres-Gordillo, García-Jiménez y Herrero-Vázquez, 2020).

Finalmente, debido a la situación causada por el COVID-19, una limitación del estudio viene por no haber podido mantener contacto con el alumnado para optar por otras técnicas de recogida de datos. Como pista de revisión, se necesitan nuevos análisis con otras muestras que profundicen en la intención emprendedora del estudiantado, con objeto de comparar la formación competencial percibida con la motivación por el autoempleo. Además, se podría analizar la iniciativa y el conocimiento del profesorado para aplicar metodologías innovadoras que utilicen la tecnología y las redes sociales orientadas al desarrollo de capacidades para el emprendimiento.

\section{Referencias bibliográficas}

Alaref, J.; Brodmann, S. y Premand, P. (2019). The Medium-Term Impact of Entrepreneurship Education on Labor Market Outcomes: Experimental Evidence from University Graduates in Tunisia. World Bank, 1-44. $<$ https://doi.org/10.1016/j.labeco.2019.101787>

Albertos, A.; Domingo, A. y Albertos, J. (2016). Estrategia docente para el desarrollo de la competencia digital en el aula universitaria: Del uso recreativo al uso formativo. Educar, 52(2), 243-261. $<$ https://doi.org/10.5565/rev/educar.732>

Bacigalupo, M.; Kampylis, P.; Punie, Y. y Van den Brande, G. (2016). EntreComp: The Entrepreneurship Competence Framework. Luxemburgo: European Commission.

Barreno, C. (2011). Zygmunt Bauman y la sociedad líquida. Esfinge (1 de septiembre). Recuperado el 5 de marzo de 2020, de <https://www.revistaesfinge.com/ filosofia/corrientes-de-pensamiento/item/757-56zygmunt-bauman-y-la-sociedadliquidas.

Becerra-Molina, E.; Sañay, I. y Calle Masache, O. (2018). Desarrollo del marketing digital para impulsar el emprendimiento, en el cantón Gualaceo-AzuayEcuador. Recimundo: Revista Científica de la Investigación y el Conocimiento, 2(4), 90-113.

$<$ https://doi.org/10.26820/recimundo/2.(4).octubre.2018.90-113> 
Calderón-Garrido, D.; León-Gómez, A. y Gil-Fernández, R. (2019). El uso de las redes sociales entre los estudiantes de Grado de Maestro en un entorno exclusivamente online. Vivat Academia, 147, 23-40. <https://doi.org/10.15178/va.2019.147.23-40>

Castro-Villacis, B.; Rodríguez-Figueroa, L.; Arroyo-Vargas, E. y ValdésCabrera, V. (2018). La importancia de las TIC en la construcción de opinión pública de los emprendimientos. INNOVA Research Journal, 3(2), 147-160. <https://doi.org/10.33890/innova.v3.n2.2018.591>

Civila-Salas, A.C. (2017). Emprendimiento, proyecto de vida y transferencia del conocimiento. En Simposio Internacional El Desafio de Emprender en la Escuela del Siglo XXI. Universidad de Sevilla.

ErdoĞmuş, C.; Korkmaz, Ö.; ÇAKIr, R. y UĞUr-erdoĞmuş, F. (2019). The Examination of Public Education Center Trainers' Attitudes towards Social Media and Their Self-Efficacy in Social Networks for Instructional Purposes. Participatory Educational Research, 6(1), 54-69. $<$ https://doi.org/10.17275/per.19.5.6.1>

Estévez-Gualda, J. y García-Marín, A. (2015). Las Redes Sociales para la mejora de la capacidad de emprender y de autoempleo. IJERI: International Journal of Educational Research and Innovation, 4, 101-110. Recuperado el 11 de marzo de 2020, de <https:/www.upo.es/revistas/index.php/IJERI/article/view/1462>.

Estrada de la Cruz, M.; Mira-Solves, I. y Gómez-Gras, J.M. (2016). El entorno universitario como generador de capacidades emprendedoras: Un análisis de percepciones. En XIV Jornadas de Redes de Investigación en Docencia Universitaria: Investigación, innovación y enseñanza universitaria: enfoques pluridisciplinares, 29262946. Recuperado el 11 de marzo de 2020, de <https://web.ua.es/es/ice/jornadasredes-2016/documentos/tema-4/806575.pdf>.

European Commission (2016). Entrepreneurship education at school in Europe. Luxemburgo: Publication Office of the European Union, 10, 593884.

Fernández-Ferrer, M. y Forés-Miravalles, A. (2018). Evaluación del desarrollo competencial en la educación superior: La perspectiva del profesorado universitario. Educar, 54(2), 391-410. $<$ https://doi.org/10.5565/rev/educar.799>

Fernández-Trigueros, F. y Fernández-Castaño, F. (2014). El auge de los emprendedores: Análisis de su presencia en prensa en 2011-2013 y en redes sociales. Vivat Academia, 127, 117-155. <https://doi.org/10.15178/va.2014.127.117-155>

Fuster-Gullén, D.E.; Serrato-Cherres, A.; Gonzales-Álvarez, R.; GoicocheaEuribe, N.F. y Guillén-Aparicio, P.E. (2020). Uso de redes sociales en el desarrollo de estrategias de lectura crítica hipertextual en estudiantes universitarios. Propósitos y Representaciones, 8(1). <https://doi.org/10.20511/pyr2020.v8n1.432>

GEM (Global EnTrepreneurship Monitor) (2017-2018). Informe GEM España. Recuperado el 11 de marzo de 2020, de <https://www.creaciondempresas.es/preparate_para_emprender/decision-de-emprender/quien-emprende-espana/>.

Gómez-NúNÉEz, L.; Llanos-Martínez, M.; Hernández-Rico, T.; MejÍa-Rodríguez, D.; Heilbron-López, J.; Martín-Gallego, J.; Mendoza-Soto, J. y Senior-Roca, D. (2017). Competencias emprendedoras en Básica Primaria: Hacia una educación para el emprendimiento. Pensamiento \& Gestión, 43, 150-188. Recuperado de <https://rcientificas.uninorte.edu.co/index.php/pensamiento/article/view/9793>. 
Gutiérrez-Olvera, S. (2015). Emprendimiento en las empresas familiares. Revista Iberoamericana de Contaduría, Economía y Administración: RICEA, 4(7), 163-181. <https://doi.org/10.23913/ricea.v4i7.119>

Herrero-Vázquez, E.-A. y Torres-Gordillo, J.-J. (2020). ECO en la Educación Superior: Un aprendizaje resonante. En M. Reyes-Tejedor, D. Coвos-Sanchiz y E. LóPEZ-MENESES (coords.). Innovación pedagógica universitaria: Reflexiones y estrategias (pp. 133-153). Barcelona: Octaedro.

Jiménez-Marín, G.; Elías-Zambrano, R. y Silva-Robles, C. (2014). Innovación docente y su aplicación al EEES. Historia y Comunicación Social, 19(3), 187-196. $<$ https://doi.org/10.5209/rev_HICS.2014.v19.45125>

Justiniano-Domínguez, M. (2019). Las trayectorias profesionales de los estudiantes de máster en Educación: Un análisis desde las expectativas y la transición. Educar, 55(2), 419-434. <https://doi.org/10.5565/rev/educar.1040>

Melero-Aguilar, N.; Torres-Gordillo, J.-J. y García-JimÉnez, J. (2020). Retos del profesorado universitario en el proceso de enseñanza-aprendizaje: Aportaciones del método ECO (Explorar, Crear y Ofrecer). Formación Universitaria, 13(3), 157-168. <https://doi.org/10.4067/S0718-50062020000300157>

Menna, A.; Catalfamo, H. y Girolamo, C. (2016). Applied Entrepreneurship Policy: Ontario's Colleges in the Age of Globalization. Educational Planning, 23(2), 59-74. Recuperado el 4 de abril de 2020, de <https://files.eric.ed.gov/fulltext/ EJ1208258.pdf>.

Mese, C. y Aydin, G.S. (2019). The Use of Social Networks among University Students. Educational Research and Reviews, 14(6), 190-199. $<$ https://doi.org/10.5897/ERR2018.3654>

Mirembe, D.P.; Lubega, J.T. y Kibukamusoke, M. (2019). Leveraging Social Media in Higher Education: A Case of Universities in Uganda. European Journal of Open, Distance and E-Learning, 22(1), 70-84. $<$ https://doi.org/10.2478/eurodl-2019-0005>

Morales, A. y Corredor, H.A. (2016). Las redes sociales: Una estrategia pedagógica para incentivar el emprendimiento. Ciencia y Poder Aéreo, 11(1), 242-255. <https://doi.org/10.18667/cienciaypoderaereo.522>

Oleforo-Ngozika A.; Око-Dominic, E. у Aкpan, E.G. (2013). Entrepreneurship Training Programme in Universities and Graduates' Productivity in South-South Nigeria. International Education Studies, 6(7), 260-266. $<$ https://doi.org/10.5539/ies.v6n7p260>

Ortiz-García, P. y Olaz-Capitán, A.J. (2015). Competencias y emprendimiento desde la perspectiva de los jóvenes. Revista Iberoamericana de Relaciones Laborales, 31-32, 17-36. <https://doi.org/10.33776/trabajo.v0i31/32.2812>

Palos-Sánchez, P.R.; Baena-Luna, P. y Casablanca-Peña, A. (2019). Análisis de las competencias educativas para evaluar a las personas emprendedoras. Interciencia: Revista de Ciencia y Tecnología de América, 44(5), 291-297. Recuperado de $<$ http://hdl.handle.net/10433/6569>.

Peñarroya-Farell, M. (2014). Las oportunidades de las redes sociales para las pymes. Oikonomics: Revista de Economía, Empresa y Sociedad, 2, 20-24. Recuperado el 16 de marzo de 2020, de <http://oikonomics.uoc.edu/divulgacio/oikono mics/_recursos/documents/02/Oikonomics_MPenarroya_es.pdfs. 
Qiao, X. y HuA, J.H. (2019). Effect of College Students' Entrepreneurial Self-Efficacy on Entrepreneurial Intention: Career Adaptability as a Mediating Variable. International Journal of Educational Methodology, 5(3), 305-313. $<$ https://doi.org/10.12973/ijem.5.3.305>

RodríGUez-AcostA, B.M. (2018). Los negocios realizados por internet: ¿Cambian los paradigmas existentes? IUS: Revista del Instituto de Ciencias Jurídicas de Puebla, 12(41), 71-85. $<$ https://doi.org/10.35487/rius.v12i41.2018.353>

SÁnchez-García, M.F. (2017). Orientación para el desarrollo profesional. Madrid: UNED.

Torres-Briones, R.M.; Espinoza-Briones, H.B.; Macías-Pettao, R.K.; ChávezPirca, C.E.; Fajardo-Arriaga, J. y Martínez-Ortiz, F.X. (2018). Interacción universidad-entorno: Una mirada para el emprendimiento en el siglo xxi.Didasc@ lia: Didáctica y Educación, 9(2), 1-16. $<$ https://doi.org/10.5294/edu.2015.18.1.7>

Torres-Coronas, T.; Vidal-Blasco, M.A. y Arias-Oliva, M. (2014). E-emprendimiento en la Educación Superior: La competencia digital. Revista Iberoamericana de Educación, 64(2). $<$ https://doi.org/10.35362/rie642360>

Torres-Gordillo, J.-J.; García-Jiménez, J. y Herrero-VÁzquez, E.-A. (2020). Contributions of technology to cooperative work for university innovation with Design Thinking. Pixel-Bit: Revista de Medios y Educación, 59, 27-64. $<$ https://doi.org/10.12795/pixelbit.74554>

Torres-Gordillo, J.-J. y Herrero-VÁzquez, E.-A. (2020). Innovación metodológica transdisciplinar en la universidad con el método ECO. En M. Reyes-TejeDOR, D. Cobos-SANChiz y E. López-Meneses (coords.). Innovación pedagógica universitaria: Reflexiones y estrategias (pp. 155-173). Barcelona: Octaedro.

Torres-Gordillo, J.-J.; Melero-Aguilar, N. y García-Jiménez, J. (2020). Improving the university teaching-learning process with ECO methodology: Teachers' perceptions. PloS One, 15(8), 1-14. e0237712. $<$ https://doi.org/10.1371/journal.pone.0237712>

Trejo-López, E.A. (2016). Perfil psicosocial de la persona emprendedora. Universidad de Valladolid, España. Tesis doctoral.

Zenner, L.; Kothandaraman, K. y Pilz, M. (2017). Entrepreneurship Education at Indian Industrial Training Institutes: A Case Study of the Prescribed, Adopted and Enacted Curriculum in and around Bangalore. International Journal for Research in Vocational Education and Training, 4(1), 69-94.

<https://doi.org/10.13152/IJRVET.4.1.4> 